\title{
Intrinsically disordered proteins in biology: One for all, all for one
}

\section{A R T I C L E I N F O}

\section{Keywords:}

Algae

Biophysical techniques

Fuzzy complexes

Intrinsic disordered protein

Kinetics

Plant

Virus

Proteins in solution are present in different conformations, which are restricted, in most cases, to local conformational changes in dynamic equilibria [1]. Intrinsic disordered proteins (IDPs), on the other hand, have a large conformational heterogeneity, formed by diverse ensembles of structures sampling a wide range of timescales [2,3]. In the fastest dynamics (nanosecond to microsecond range), polypeptide chain reconfiguration occurs [4]; flickering secondary structure formation happens in the microsecond range; and changes in more persistent interactions (usually involving long-range contacts) take place in the millisecond time scale [5,6]. All these movements of IDPs are usually faster than the kinetics of the binding reactions to their natural partners [6,7]. In fact, their high flexibility and the absence of a wellfixed structure allow IDPs to: (i) exploit unique protein-macromolecule interactions, and (ii) carry out biological functions involving different partners [8]. In this sense, IDPs behave as one of the three musketeers [9], acting an isolated IDP in concert with other - in some cases, many, as happens in the hubs - proteins to modulate complex interaction networks (one for all) [10]. This binding occurs by selecting a particular conformation of the whole IDP ensemble, and shifting the conformational equilibrium of the whole ensemble (all for one) to the bindingprone conformation with the corresponding partner [10-12].

In this Special Issue on IDPs, we address their importance in biology in the last 10 years. The first five manuscripts encompass the use of several experimental equilibrium and kinetic techniques, together with a theoretical, physical point of view in describing IDPs. The next manuscripts review: (i) the fuzziness observed intramolecularly for some multi-domain proteins; (ii) how complexity and flexibility can also modulate the architecture of membrane proteins; and (iii) the presence and importance of IDPs in several kingdoms of life.

To study the structural features of IDPs, we now have a plethora of biophysical techniques. Longhi and co-workers describe in their extensive review most of the techniques currently used to address the structural and conformational features of IDPs either in vitro or in vivo [13]. For instance, they describe the information provided by spectroscopic techniques such as circular dichroism (CD) and infra-red (FTIR), which, by using deconvolution methods, provide hints on the local structure present in IDPs. Furthermore, the authors address use in vivo of other spectroscopic techniques that describe how the possible crowding effect, within cells, can shift the ensemble of IDP conformations. The review finishes with an introduction to recent computational techniques used to describe that ensemble with the concomitant employment of experimental information.

However, experimental atomic resolution data of the conformational ensemble can be only provided by high-resolution techniques such as nuclear magnetic resonance (NMR). Instead of describing the recently developed methods of assigning IDPs by NMR, Assfalg and coworkers focus on recent applications of NMR to tackle supramolecular assemblies of IDPs involved in neurological diseases [14]. In recent years, membrane-less organelles have been observed to form by liquidliquid phase separation (LLPS) $[15,16]$. These organelles are, in many cases, formed by IDPs, or alternatively, by proteins that contain disordered regions (IDRs). The conformational study of these organelles has paved the way to study oligomerization and supra-macromolecular assembly of several aggregation-prone proteins. Assfalg and colleagues describe first the NMR approaches to probe the conformations of IDPs forming those assemblies, as a result of aggregation. As examples, they describe early-stage oligomerization and intermolecular interactions of synucleins and huntingtin and molecular exchange reactions on the surface of amyloid $\beta$-protofibrils formed by the $\beta$-amyloid polypeptide. At the end, the authors describe the LPPS occurring in Tau protein and

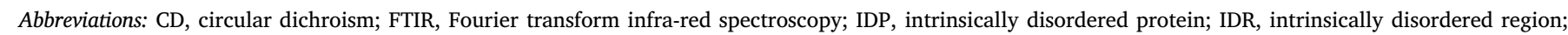

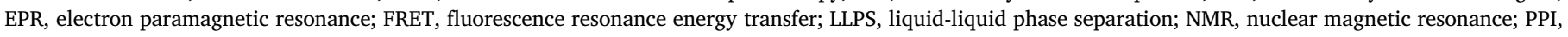
protein-protein interactions; SAXS, small angle X-ray scattering 
in the fused in sarcoma protein. In fact, both phenomena (aggregation and LPPS) are the result of having high protein concentrations, where intermolecular interactions start competing with the initial disordered state of IDPs. The review is a tour through state-of-the-art NMR techniques describing invisible (and yet observable) states in those supramacromolecular assemblies.

Atomic-resolution information is not only provided by NMR, but also by electron paramagnetic resonance (EPR). As it happens with her sister (NMR), it measures the spectroscopic resonance of spin changes in electrons, instead of protons in the nucleus [17]. In combination with specific spin-labeling at positions designed by site-directed mutagenesis, EPR provides structural and dynamic information on IDPs. The technique provides complementary information to that of NMR concerning the different correlation times explored by proteins and IDPs. García-Rubio [18] describes how the introduction of several probes in different positions allows one to obtain reliable distributions of distances in an IDP and how its flexibility and the population of conformers is shifted upon binding to its natural partners. The review can be also used as a general introduction for those hoping to use the benefits of the technique for well-folded proteins.

The above techniques can provide dynamic information, but that comes from the exploration of the different populated conformations (when either the IDP is free or bound). However, we do not have information on the kinetics of the proper binding process to the natural partners of an IDP. That information can be only obtained by kinetic (stopped-flow techniques, among others [19]) methods. Gianni and colleagues describe [20] how the kinetic techniques, under different conditions, can be exploited to describe the conformational landscape of an IDP and its hot-spot regions (i.e., the polypeptide patches involved in the binding). Their review is an excellent compilation of our current knowledge of the kinetic mechanisms of several binding reactions involving IDPs.

The application of many of the results provided by the above experimental techniques can be only understood within the framework of the theory of polymers and polyelectrolytes developed in the $\mathrm{XX}^{\text {th }}$ century [1]. The polymeric nature of protein chains must be taken into account when we are trying to understand the features of IDPs, and the study of isolated residues or protein fragments of the IDPs may not provide enough clues [21]. In fact, the conformational properties of an IDP are ruled by an exquisite balance among solvent-solvent, solventchain and chain-chain interactions, which can be only understood when the whole polypeptide chain is studied. Soranno describes, by using mathematical language, how to interpret and explain the experimental measurements obtained, for instance, for the radius of gyration in IDPs, measured by small angle X-ray Scattering (SAXS) and fluorescence resonance energy transfer (FRET), within the theory of disordered polymers [22] and how this size is changed in the disorder-to-order transition occurring when most of IDPs bind to their natural partners. The review contains an in-depth description of the physics of polymers, easily understood by anyone interested in knowing how disordered chains behave in the presence of different solvents.

When some IDPs bind to their partners, they keep their flexibility: this is the concept of "fuzzy" complexes, where at least one of the members of the complex maintains its flexibility [23,24]. Arbesus and Pons describe the presence of these "fuzzy" interactions for a multimodular protein: the Src family of kinases, which contains both ordered and non-ordered regions [25]. In this case, the "fuzzy" contacts occur intramolecularly between a well-folded module of the Src protein (a SH3 domain) and the disordered N-terminal region (module) of the same protein, containing multiple phosphorylation sites. Based on their own model and those of other researchers, the authors conclude that these intramolecular fuzzy complexes can be conserved through evolution if they are involved in key functional interactions. They further suggest that SH3 domains can be considered as the receptor gates for IDRs, acting as connectors between the globular, well-folded actuators and the IDPs sensors.
Usually membrane proteins are not described in Special Issues dedicated to IDPs, because membrane proteins are well-folded proteins embedded with a lipid environment. However, it has been estimated that $40-50 \%$ of transmembrane proteins contain IDRs of significant length [26-28]. These IDRs, which usually occur in the intra/extra cellular loops of transmembrane proteins, can have a role in stabilizing the overall protein structure due to their large accumulation of positive charges [29]. Thus, IDPs are not only involved in the formation of membrane-less organelles [14], but also they intervene in defining the structural features of membrane proteins. Claessens and colleagues discuss in their review [30] how membrane-bound IDPs or, alternatively, IDRs can: (i) modulate the membrane curvature; (ii) join membranes together; and (iii) modify local lipid composition.

There is a low proportion of IDPs or alternatively IDRs in bacteria and archaea, a higher one in viruses, and a much larger one in eukaryotes (although in some viruses the proportion of IDPs can be similar to that in eukaryotes) [31,32]. Proteins of viruses are involved in many protein-protein interactions (PPI) to: (i) activate the host cell machinery; (ii) enhance virulence; and (iii) interfere with the host immune response. Therefore, it is not surprising that the proteomes of many viruses have a large amount of IDPs or IDRs [8,31]. The Flaviviridae taxon consists of a large family of viral pathogens involved in several human diseases [33]. Flaviviruses have small proteomes but a large interactome. Santos and Martins describe our current knowledge on the disorder proteome of several flavivirus [34] including various examples of IDPs involved in the capsid assembly and the features of non-structural proteins (NS4 and NS2).

Because of their large flexibility, IDPs are capable of maintaining their functional states under a wide range of conditions, even under the harsh ones faced by some algae. Gontero and colleagues describe several examples of IDPs either found by sequence predictions or structurally characterized by using several biophysical techniques on algae in vitro [35]. They focus on examples from IDPs involved in algal metabolism. Especially interesting is the case of CP12, a chloroplastic protein involved in the Calvin-Benson cycle, which upon binding to other well-folded protein not only remains partially unfolded (as in the "fuzzy" complexes described above [25]), but even unfolds to a larger extent than when it is isolated. This process, called "cryptic disorder", reduces the entropy cost of binding; it has only been reported in a few examples.

The inherent flexibility of IDPs allows the immediate modulation of cellular conditions to the environmental changing situations suffered by plants. As in other eukaryotes, in plants IDPs act as hubs in protein networks - signalling, cell cycle, stress response (including the immune one) and development - capable of binding to different partners, and forming the scaffold for higher order complexes. Covarrubias and colleagues describe the presence of IDPs in some of these networks (signalling, metabolism and stress responses) in plants [36]. Some examples belong to the transmembrane proteins with IDRs as those described before [30], and they even illustrate the presence of LLPSs orchestrated by IDPs in several plant processes. Their manuscript is an enlightening description of several regulatory pathways in plants, and how similar some of them (e.g., signalling) are to those found in other eukaryotes.

Each IDP is unique in its flexibility and in its particular (local and long-range) features, but in all the examples described in this Special Issue the common features of large flexibility and involvement in protein networks appear (one for all); therefore, all the knowledge acquired by studying several IDPs can be applied, at least partially, to understand better a particular one (all for one). The continuing technological innovations in the techniques currently used, and the development of new software to describe the conformational ensemble of a particular IDP will have an increasingly key role in studies of biological systems in the years to come, and probably in the development of new drugs and phytochemicals.

As editors, we would like to close this introduction by 
acknowledging that while we were fortunate to showcase some of the most important advances that have occurred in the field of IDPs in the past 10 years, we were by no means able to present all of them. Accordingly, we apologize in advance to our colleagues and friends whose work we were unable to include as part of this Special Issue. Additionally, we would like to offer our gratitude to the authors and reviewers and to Prof. Paul Fitzpatrick for his comments on this Introduction, as well as editorial staff of Archives of Biochemistry and Biophysics (Divya Pillai and Claire Brimilcombe), whose expertise and dedication to this topic made this Special Issue possible.

\section{References}

[1] K.A. Dill, S. Bromberg, Molecular Driving Forces: Statistical Thermodynamics in Biology, Chemistry, Physics and Nanosciences, second ed., Garland Science, USA, 2011.

[2] N. Salvi, A. Abyzov, M. Blackledge, Atomic resolution conformational dynamics of intrinsically disordered proteins from NMR relaxation, Prog. Nucl. Magn. Reson. Spectrosc. 102-103 (2017) 43-60.

[3] R.B. Best, Computational and theoretical advances in studies of intrinsically disordered proteins, Curr. Opin. Struct. Biol. 42 (2017) 147-154.

[4] M. Brucale, B. Schuler, B. Samori, Single-molecule studies of intrinsically disordered proteins, Chem. Rev. 114 (2014) 3281-3317.

[5] R.B. Best, Emerging consensus on the collapse of unfolded and intrinsically disordered proteins in wáter, Curr. Opin. Struct. Biol. 60 (2020) 27-38.

[6] B. Schuler, A. Borgia, M.B. Borgia, P.O. Heidarsson, E.D. Holmstrom, D. Nettels, A. Sottini, Binding without folding-the biomolecular function of disordered polyelectrolyte complexes, Curr. Opin. Struct. Biol. 60 (2020) 66-76.

[7] S.L. Shammas, M.D. Crabtree, L. Dahal, B.I. Wicky, J. Clarke, Insights into coupled folded and binding mechanism from kinetic studies, J. Biol. Chem. 291 (2016) $6689-6695$

[8] V.N. Uversky, Intrinsically disordered proteins and their "mysterious" (meta)physics, Front. Phys. 7 (2019) 10.

[9] A. Dumas, Les Trois Mousquetaires. La Siècle Newspaper (France)1844; as a single volume by Editorial Braudy (France) 1846, (1844).

[10] P. Tompa, E. Schad, A. Tantos, L. Kalmar, Intrinsically disordered proteins: emerging interaction specialists, Curr. Opin. Struct. Biol. 35 (2015) 49-59.

[11] P.E. Wright, H.J. Dyson, Intrinsically disordered proteins in cellular signaling and regulation, Nat. Rev. Mol. Cell Biol. 16 (2015) 18-29.

[12] A. Bah, J.D. Forman-Kay, Modulation of intrinsically disordered protein function by post-translational modification, J. Biol. Chem. 291 (2016) 6696-6705.

[13] A. Schramm, C. Bignon, S. Brocca, R. Grandori, C. Santambrogio, S. Longhi, An arsenal of methods for the experimental characterization of intrinsically disordered proteins - how to choose and combine them? Arch. Biochem. Biophys. 676 (2019), https://doi.org/10.1016/j.abb.2019.07.020 108055.

[14] F. Munari, M. D'Onofrio, M. Assfalg, Solution NMR insights into dynamic supramolecular assemblies of disordered amyloidogenic proteins, Arch. Biochem. Biophys. (2020).

[15] Y. Shin, Y.C. Chang, D.S.W. Lee, J. Berry, D.W. Sanders, P. Ronceray, N.S. Wingreen, M. Haataja, C.P. Brangwynne, Liquid nuclear condensates mechanically sense and restructure the genome, Cell 176 (2019) 1518, https://doi. org/10.1016/j.cell.2019.02.025.

[16] D. Bracha, M.T. Walls, C.P. Brangwynne, Probing and engineering liquid phase organelles, Nat. Biotechnol. 37 (2019) 1435-1445, https://doi.org/10.1038/ s41587-019-0341-6.

[17] R. Guzzi, R. Bartucci, Electron spin resonance of spin-labeled lipid assemblies and proteins, Arch. Biochem. Biophys. 580 (2015) 102-111.

[18] I. García-Rubio, EPR of site-directed spin-labeled proteins: a powerful tool to study structural flexibility, Arch. Biochem. Biophys. (2020).

[19] A.R. Fersht, Structure Mechanism and Protein Science, W.H Freeman, USA, 1999.

[20] A. Toto, F. Troilo, L. Visconti, F. Malagrinò, C. Bignon, S. Longhi, S. Gianni, Binding induced folding: lessons from the kinetics of interaction between NTAIL and XD, Arch. Biochem. Biophys. 671 (2019) 255-261, https://doi.org/10.1016/j.abb. 2019.07.011.

[21] R.V. Pappu, X. Wang, A. Vitalis, S.L. Crick, A polymer physics perspective on driving forces and mechanisms for protein aggregation, Arch. Biochem. Biophys 469 (2008) 132-141.

[22] A. Soranno, Physical basis of the disorder-order transition, Arch. Biochem. Biophys. (2020).

[23] P. Tompa, M. Fuxreiter, Fuzzy complexes: polymorphism and structural disorder in protein-protein interactions, Trends Biochem. Sci. 33 (2008) 2-8.

[24] M. Miskei, C. Antal, M. Fuxreiter, FuzDB: database of fuzzy complexes, a tool no develop stochastic structure-function relationships for protein complexes and higher order assemblies, Nucleic Acids Res. 45 (2017) D228-D234.

[25] M. Arbesu, M. Pons, Interacting disorder in globular multidomain proteins: fuzzy sensors and the role of SH3 domains, Arch. Biochem. Biophys. 677 (2019) 108161, https://doi.org/10.1016/j.abb.2019.108161.

[26] Y. Minezaki, K. Homma, K. Nishikawa, Intrinsically disordered regions of human plasma membrane proteins preferentially occur in the cytoplasmic segment, J. Mol. Biol. 368 (2007) 902-913.

[27] A. Tantos, K.H. Han, P. Tompa, Intrinsic disorder in cell signaling and gene transcription, Mol. Cell. Endocrinol. 348 (2012) 457-465.

[28] J. Burgi, B. Xue, V.N. Uversky, F.G. van der Groot, Intrinsic disorder in transmembrane proteins: roles in signaling and topology prediction, Plos One 1 (2016) e0158594.

[29] G.E. Tusnady, L. Dobson, P. Tompa, Disordered regions in transmembrane proteins, Biochim. Biophys. Acta Biomembr. 1848 (2015) 2839-2848.

[30] M.A. Fakhree, C. Blum, M.M.A.E. Claessens, Shaping membranes with disordered proteins, Arch. Biochem. Biophys. 677 (2019), https://doi.org/10.1016/j.abb. 2019.108163108163.

[31] G. Goh, A.K. Dunker, V.N. Uversky, Protein intrinsic disorder toolbox for comparative analysis of viral proteins, BMC Genom. 9 (2008), https://doi.org/10.1186/ 1471-2164-9-S2-S4 S4.

[32] Z. Peng, J. Yan, X. Fan, M.J. Mizianty, B. Xue, J. Wang, G. Hu, V.N. Uversky, Exceptionally abundant exceptions: comprehensive characterization of intrinsic disorder in all domains of life, Cell. Mol. Life Sci. 72 (2015) 137-151.

[33] S. Mukhopadhyaya, R.J. Kuhn, M.G. Rossman, A structural perspective of the flavivirus life cycle, Nat. Rev. Microbiol. 13 (2005), https://doi.org/10.1038/ nrmicro1067.

[34] I.C. Martins, N.C. Santos, Intrinsically disordered proteins in Flavivirus infection, Arch. Biochem. Biophys. (2020), https://doi.org/10.1016/j.abb.2020.108298 108298.

[35] H. Lunay, V. Receveur-Bréchot, F. Carrière, B. Gontero, Orchestration of algal metabolism by protein disorder, Arch. Biochem. Biophys. (2019), https://doi.org/ 10.1016/j.abb.2019.108070108070.

[36] A.A. Covarrubias, P.S. Romero-Pérez, C.L. Cuevas-Vázquez, D.F. Rendón-Luna, The functional diversity of structural in plant proteins, Arch. Biochem. Biophys. (2019), https://doi.org/10.1016/j.abb.2019.108229 108229.

Inmaculada Yruela*

Group of Computational and Structural Biology, Estación Experimental de Aula Dei (EEAD-CSIC), Avda. Montañana 1005, 50059, Zaragoza, Spain Group of Biochemistry, Biophysics and Computational Biology (BIFI-

Unizar) Joint Unit to CSIC, Spain

E-mail addresses: i.yruela@csic.es, yruela@eead.csic.es.

José L. Neira***

Instituto de Biología Molecular y Celular, Universidad Miguel Hernández, 03202, Elche, Alicante, Spain

Instituto de Biocomputación y Física de Sistemas Complejos (BIFI), Universidad de Zaragoza, 50018, Zaragoza, Spain E-mail address: jlneira@umh.es.

\footnotetext{
${ }^{*}$ Corresponding author. Estación Experimental de Aula Dei (EEAD-CSIC). Avda. Montañana 1005, 50059, Zaragoza, Spain.

${ }^{* *}$ Corresponding author. Instituto de Biología Molecular y Celular, Universidad Miguel Hernández, Avda. del Ferrocarril s/n, 03202, Elche, Alicante, Spain.
} 